\title{
Crimean-Congo hemorrhagic fever virus in livestock ticks and animal handler seroprevalence at an abattoir in Ghana
}

\author{
R. Akuffo ${ }^{1,3,6^{*}}$, J. A. M. Brandful ${ }^{3}$, A. Zayed ${ }^{1}$, A. Adjei ${ }^{4}$, N. Watany ${ }^{1}$, N. T. Fahmy ${ }^{1}$, R. Hughes ${ }^{1}$, B. Doman',
} S. V. Voegborlo' ${ }^{1}$ D. Aziati ${ }^{3}$, D. Pratt ${ }^{3}$, J. A. Awuni ${ }^{5},{ }^{2}$. Adams ${ }^{1}$ and E. Dueger ${ }^{1,2}$

\begin{abstract}
Background: Crimean-Congo Haemorrhagic Fever Virus (CCHFV) is a zoonotic virus transmitted by Ixodid ticks and causes Crimean-Congo hemorrhagic fever (CCHF) disease in humans with up to $50 \%$ mortality rate.

Methods: Freshly slaughtered livestock at the Kumasi abattoir in the Ashanti Region of Ghana were examined for the presence of ticks once a month over a 6-month period from May to November 2011. The ticks were grouped into pools by species, sex, and animal source. CCHFV was detected in the ticks using reverse transcription PCR. Blood samples were collected from enrolled abattoir workers at initiation, and from those who reported fever in a preceding 30-day period during monthly visits $2-5$ months after initiation. Six months after initiation, all participants who provided baseline samples were invited to provide blood samples. Serology was performed using enzyme linked immunosorbent assay (ELISA). Demographic and epidemiological data was also obtained from enrolled participants using a structured questionnaire.

Results: Of 428 freshly slaughtered animals comprising 130 sheep, 149 cattle, and 149 goats examined, 144 ticks belonging to the genera Ambylomma, Hyalomma and Boophilus were identified from 57 (13.3 \%): 52 (34.9\%), 4 (3.1 \%) and $1(0.7 \%)$ cattle, sheep and goat respectively. Of 97 tick pools tested, 5 pools comprising 1 pool of Hyalomma excavatum and 4 pools of Ambylomma variegatum, collected from cattle, were positive for CCHFV. Of 188 human serum samples collected from 108 abattoir workers, 7 (3.7 \%) samples from 6 persons were anti-CCHF IgG positive with one of them also being CCHF IgM positive. The seroprevalence of CCHFV identified in this study was $5.7 \%$.
\end{abstract}

Conclusions: This study detected human exposure to CCHF virus in slaughterhouse workers and also identified the CCHF virus in proven vectors (ticks) of Crimean Congo hemorrhagic fever in Ghana. The CCHFV was detected only in ticks collected from cattle, one of the livestock known to play a role in the amplification of the CCHF virus.

Keywords: Crimean-congo hemorrhagic fever virus, Crimean-congo hemorrhagic fever, Seroprevalence, Ticks, Ambylomma, Hyalomma, Boophilus, Livestock, Abattoir, Ghana

\section{Background}

Crimean-Congo Haemorrhagic Fever Virus (CCHFV) is a member of the Nairovirus genus of the family Bunyaviridae that can be transmitted to humans. This arbovirus is transmitted by Ixodid ticks and causes a highly pathogenic disease called Crimean Congo hemorrhagic fever (CCHF) with up to $50 \%$ mortality rate in humans

\footnotetext{
* Correspondence: Richard.akuffo@gmail.com; rakuffo@ug.edu.gh

${ }^{1}$ U.S Naval Medical Research Unit No. 3, Cairo, Egypt

${ }^{3}$ Noguchi Memorial Institute for Medical Research, University of Ghana, Accra, Ghana

Full list of author information is available at the end of the article
}

[1, 2]. At least 28 species of the Ixodid ticks distributed among 7 genera, (Hyalomma, Rhipicephalus, Boophilus, Dermacentor, Ambylomma, Haemaphysalis, and Ixodes) have been found to be naturally infected with $\mathrm{CCHF}$ virus worldwide [3, 4].

Crimean Congo hemorrhagic fever (CCHF) is endemic to Africa, the Balkans, the Middle East, and parts of Asia. Although the hard ticks (Ixodids) serve as reservoirs and vectors for CCHFV, a variety of animals, such as cattle, sheep, goat and camels, are considered amplifying hosts for the virus. The CCHF virus has been 
isolated from ticks, domestic and wild vertebrates, and humans in some sub-Saharan West African countries such as Senegal, Mauritania, and Burkina Faso [5-8].

The CCHFV can cause a severe disease in humans by exposure to bites from infected ticks or by crushing infected ticks with an open wound, contact with blood or tissues from infected patients and animals, and drinking unpasteurized milk from infected animals. Other documented modes of transmission include aerosol transmission as well as horizontal transmission from a mother to her child in Russia. However, the virus causes limited or no disease in their zoonotic hosts $[9,10]$.

Several factors influence CCHF morbidity and mortality. The incidence of the CCHF disease as well as the numbers of ticks in the environment is influenced by climatic factors, geographic conditions and the presence and habitat preferences of host animals. As a result, seasonality of the disease has been observed in some countries such as Iran and Pakistan [11, 12]. In addition, reports from about 30 countries in Africa, Middle East and Asia have identified a relationship between the geographic pattern of distribution of the CCHF disease and the distribution of Hyalomma tick vectors [13].

Furthermore, most CCHF cases are the result of occupational exposure among abattoir workers, farmers, shepherds, veterinarians, laboratory workers and healthcare workers. During a nosocomial outbreak at a hospital in South Africa, 33 \% of medical personnel exposed via needle stick injuries became ill while approximately $9 \%$ of those who had other forms of contact with infected blood also developed CCHF [14].

Previous CCHF seroprevalence studies among pastoralists, healthy populations in endemic communities as well as in outpatient settings have provided valuable information on the disease epidemiology including risk factors and hot spots which have enabled a more focused preventive approach towards limiting the spread of this disease in many countries [10, 15]. As a result, surveillance for CCHFV in human and vector populations provides an opportunity to monitor the likelihood of a disease of potentially severe impact in humans [16].

As part of a broader study examining occupational risk of exposure to vector-borne and zoonotic pathogens in a high-risk abattoir worker population in Ghana, hard ticks were collected from freshly slaughtered livestock and tested for CCHFV. In addition, human blood samples were collected and tested for CCHF virus specific IgM and IgG antibodies.

\section{Methods}

Samples were collected once a month over a 6-month period at the Kumasi Abattoir in the Ashanti Region of Ghana, spanning May to November 2011.
Freshly slaughtered animals including cattle, goat and sheep were examined for the presence of hard ticks. Ticks were removed from the freshly slaughtered animals, using blunt forceps and placed in cryogenic vials containing RNAlater solution to maintain intact RNA during shipment.

All samples were transported to US Naval Medical Research Unit No. 3 in Cairo, Egypt, for taxonomic identification using the African Ixodidae key [3]. After identification, the ticks were grouped into pools by species, sex, and animal source. Ticks pools with extraction Lysis buffer and specific beads were homogenized by shaking in Mini-Beadbeater-96 (BioSpec, Bartlesville, OK, USA). RNA was extracted following QIAamp Viral RNA Kit (Qiagen, Valencia, CA) protocol [17]. CCHFV $\mathrm{S}$ segment was detected by specific primers and MGB probe established by Garrison et al. [18] Reverse transcription PCR was performed using SuperScript ${ }^{\circ}$ OneStep RT-PCR System with Platinum ${ }^{\circ}$ Taq DNA Polymerase (Invitrogen, Carlsbad, CA, USA).

Animal handlers who provided consent to participate in the study were asked to provide a baseline blood sample at the first monthly visit. During monthly visits from 2 to 5 months following initial sampling, blood samples were obtained from animal handlers who reported fever during the preceding 30 days. At 6 months following study initiation, all animal handlers who provided baseline blood sample were invited to provide a follow-up blood sample.

The blood samples collected were tested for a panel of vector borne and zoonotic pathogens including CCHF, by serology at the Department of Virology, Noguchi Memorial Institute for Medical Research and confirmed at the virology department of the US Naval Medical Research Unit No. 3, Cairo, Egypt.

The CCHF virus specific IgM and IgG antibodies test was performed using a commercially available kit, which came with its own internal negative and positive controls (VectoCrimean-CHF-IgG and IgM ELISA test kits; Vector-Best, Novosobirsk, Russia). Demographic and epidemiological data were obtained from enrolled participants using a structured questionnaire.

Data was managed using Microsoft Access program and analyzed using the Statistical Package for Social Sciences (SPSS) version 20. All statistical tests were conducted at a $95 \%$ confidence level.

\section{Results}

A total of 428 freshly slaughtered animals were examined: 149 (34.8\%) cattle, 149 (34.8\%) goat and 130 (30.4\%) sheep. In total, 144 ticks were identified from $57(13.3 \%)$ of the animals: 52 (34.9\%) cattle, 4 (3.1\%) sheep and $1(0.7 \%)$ goat. 
Of the 52 tick laden cattle, 31 (59.6\%) were local breeds while $19(36.5 \%)$ were imported from Burkina Faso, 1 (1.9 \%) from Mali, and 1 (1.9 \%) from Cote d'Ivoire. Additionally, 3 (75\%) of the sheep were local breeds while 1 (25 \%) was imported from Burkina Faso. The only goat with ticks in our study was imported from Niger.

The ticks identified in this study were all from the family Ixodidae; majority, 93 (64.5\%), of which belonged to the genus Ambylomma. This included the single tick obtained from the goat and most $(60.8 \%$ and $88.9 \%)$ of those retrieved from cattle and sheep respectively.

In addition, 27 (18.8 \%) of the ticks belonged to the genus Hyalomma, comprising $20 \%$ of those retrieved from cattle and $10.1 \%$ of those from sheep. The remaining ticks, 24 (16.7\%), belonged to the genus Boophilus and were all collected from cattle.

Of 97 tick pools tested (144 ticks of the genera Ambylomma, Hyalomma and Boophilus), 5 pools were positive for CCHFV (Table 1). All 5 pools contained ticks collected from local cattle breeds. The composition of the 5 positive pools was as follows: One pool comprised female $H$. excavatum, 2 comprised female $A$. variegatum while 2 other pools comprised male $A$. variegatum; all proven vectors of CCHF $[1,5,6]$. All positive pools were comprised of adult ticks.

A total of 109 animal handlers provided consent to participate in the broader study examining occupational risk of exposure to vector-borne and zoonotic pathogens in the high-risk abattoir worker population in the Ashanti region of Ghana, out of which 108 (comprising 6 (5.6\%) females and 102 (94.4\%) males) provided blood sample at baseline.

Twenty (18.5\%) of the animal handlers who provided a baseline blood sample reported acute febrile illness during the follow-up period and provided blood samples. Sixty $(55.6 \%)$ of persons who provided baseline sample also provided blood sample at the final visit at 6 months.

From baseline through monthly visit 6, a total of 188 blood samples were collected and tested for CCHF by serology. Seven samples (3.7 \%) drawn from six persons were anti-CCHF IgG positive with 1 of the 7 also being anti-CCHF IgM positive. As a result the overall seroprevalence of CCHF identified was $5.7 \%$.

Table 1 Genera and species of 5 positive adult tick pools from which CCHFV was isolated

\begin{tabular}{lll}
\hline $\begin{array}{l}\text { Number of tick pools and } \\
\text { sex (M/F) of ticks in pools }\end{array}$ & $\begin{array}{l}\text { Genera and species of Ixodidae ticks } \\
\text { in various tick pools }\end{array}$ \\
\cline { 2 - 3 } & Ambylomma & Hyalomma \\
\hline $1(\mathrm{~F})$ & Nil & H. excavatum \\
$2(\mathrm{~F})$ & A. variegatum & $\mathrm{Nil}$ \\
$2(\mathrm{M})$ & A. variegatum & $\mathrm{Nil}$ \\
\hline
\end{tabular}

Of the 7 anti-CCHF IgG positive samples, 4 (including the 1 IgM positive sample) were collected at baseline while three were collected at the $6^{\text {th }}$ month of sample collection. None of the 6 persons who were anti-CCHF IgG or IgM positive reported a history of fever within a 30-day period prior to sample collection.

The serum sample of one of the three persons whose blood samples were anti-CCHF IgG positive at 6 months was also IgG positive at baseline, while serum samples from the other 2 persons tested negative to both antiCCHF IgM and IgG at baseline.

All seven anti-CCHF IgG/IgM samples were collected from 6 Ghanaian males within the age range of 35 to 48 years (average age $=39$ years). All six persons who were anti-CCHF IgG/IgM positive handled animal parts and were engaged in clean up of the abattoir as part of their work.

\section{Discussion}

Crimean Congo hemorrhagic fever is a considerable public health threat which can have significant effect on abattoir workers and healthcare personnel, especially in resource-poor countries [9]. The potential of CCHF to cause nosocomial outbreaks, and the limited options available for treatment and management of infected persons underscore the need for surveillance [10]. The detection of CCHFV in proven vectors of CCHF in our study, supports surveillance in abattoir workers who often may be exposed to infected ticks as well as blood and tissues of infected livestock.

The overall seroprevalence of CCHF IgG antibodies, which is indicative of prior exposure to $\mathrm{CCHV}$ in our study was $5.7 \%$. Serology results obtained after testing 188 blood samples from animal handlers at the abattoir suggest previous and active circulation of $\mathrm{CCHF}$ in the slaughterhouse workers.

Four of the seven anti-CCHF IgG samples identified in our study were detected at baseline while the remaining three were detected from samples collected at monthly visit 6 . The detection of CCHF IgG positives among 3 (4.4\%) of the samples collected at monthly visit 6 out of which two samples were from people whose serum samples neither tested positive for CCHF IgG nor IgM at baseline, suggest that those two persons may have had exposure to the $\mathrm{CCHF}$ virus after the baseline visit.

Although none of the 7 anti-CCHF IgG/IgM positives observed in this study reported acute febrile illness during a 30 day period prior to their sample collection dates, we are unable to conclude that $\mathrm{CCHF}$ virus is not currently causing acute febrile illness among the animal handlers; we relied on self reporting of fever among the animal handlers.

The dominant tick collected from Ghanaian cattle is the three-host $A$. variegatum, which is one of the most 
harmful ticks in Africa [19]. The fact that CCHFV positive pools are present in ticks collected from cattle must be considered a threat to human health, since pathogens can be disseminated through other hosts including wild animals and birds.

The results of this study also suggest that, while meat and meat products serve as an essential source of protein, trade in live animals, meat, and meat products can also serve as a mobile pool of diseases such as CCHF, with potentially large economic and health effects [16].

Furthermore, the influence of climatic conditions on tick activity and occurrence of CCHF have been identified in other studies. Future studies focusing on understanding the relationship between climatic conditions and the occurrence of CCHF could be helpful in the establishment of early warning systems in the surveillance of this disease [12].

In addition, as illustrated in Fig. 1, implementation of some CCHF risk mitigating measures such as the avoidance of tick bites, use of personal protective equipment and control of CCHF in animals by using acaricides in livestock production facilities could avert a CCHF outbreak [20].

\section{Conclusions}

This study detected human exposure to CCHF virus in a high risk abattoir population and also identified the CCHF virus in proven tick vectors in Ghana. The CCHFV was detected in ticks collected from cattle, one of the livestock

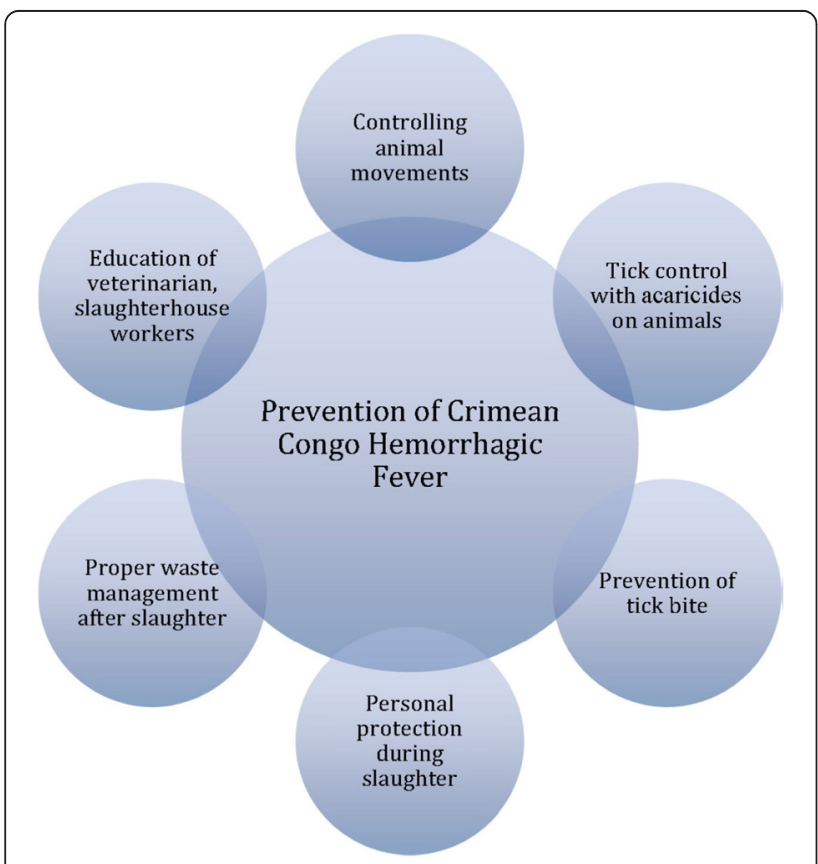

Fig. 1 Major components of prevention of Crimean-Congo Hemorrhagic Fever [20] known to play a role in the amplification of the CCHF virus.

While future studies targeted at understanding the relationship between climatic conditions and the occurrence of CCHF could be helpful in the establishment of early warning systems in the surveillance of this disease, implementation of CCHF risk mitigation measures could avert an outbreak of the CCHF disease in Ghana.

\section{Abbreviations}

CCHF, crimean-congo hemorrhagic fever; CCHFV, crimean-congo

hemorrhagic fever virus; ELISA, enzyme linked immunosorbent assay

\section{Acknowledgments}

We acknowledge management and workers from the Kumasi abattoir, Ghana, for their cooperation and support during specimen collection. The role of staff of Noguchi Memorial Institute for Medical Research in sample storage and transportation is appreciated. We also appreciate support from Ghana Veterinary Services during specimen collection. Furthermore, we recognize members of the US Naval Medical Research Unit No. 3 Vector Biology Research Program who assisted with specimen collection in Kumasi: Ms. Rania Kaldas. We also recognize members of the US Naval Medical Research Unit No. 3 Global Disease Detection and Response Program who provided project coordination support. The role of the Ghana Detachment of the US Naval Medical Research Unit No. 3 in providing logistics support to the project is also appreciated.

This project was funded by the US Department of Defense Global Emerging Infections System, work unit 847705.82000.25GB.E0018.

\section{Availability of data and materials}

Data for this manuscript as well as information on all materials used in this study are available upon request from the corresponding author. Data for this manuscript shall made available in a publicly available and accessible data repository after publishing of manuscript.

\section{Authors' contributions}

Conceived and designed the study: DE. Conducted the study: DE, BJAM, AA, VSV, AR, DB, HR, PD, FNT, ZA, AJA, AD. Analyzed the data: AR. Wrote the manuscript: ED, BJAM, AN, AA, VSV, AR, DB, HR, PD, FNT, ZA, AJA, RA, WN AD. All authors read and approved the final manuscript.

\section{Competing interests}

The authors declare that they have no competing interests.

\section{Consent for publication \\ Not applicable.}

\section{Ethics approval and consent to participate}

The study protocol was approved by the Institutional Review Boards at U.S. Naval Medical Research Unit No. 3 (NAMRU-3 IRB \# 1015), the U.S. Centers for Disease Control and Prevention (CDC IRB \# 6025), Institutional Animal Care and Use Committee, NAMRU-3 (IACUC \# NAMRU3.2010.00.15 DAU/ 1301), Noguchi Memorial Institute for Medical Research (NMIMR-IRB CPN 012/10-11) in compliance with all applicable federal regulations governing the protection of human subjects. All human participants included in the study provided consent to participate.

\section{Disclaimer}

The views expressed in this work are those of the authors and do not represent the official policy of the U.S Government, the Department of Defense or the Department of the Navy. The study protocol number 1015 entitled 'Pilot study for Integrated Human-Animal-Vector surveillance for Endemic and Emerging Vector-Borne and Zoonotic pathogens in targeted high-risk populations in Ghana' was reviewed and approved by the institutional review board of the U.S Naval Medical Research Unit No.3, in compliance with all federal regulations governing the protection of human subjects. 


\section{Author details}

${ }^{1}$ U.S Naval Medical Research Unit No. 3, Cairo, Egypt. ${ }^{2}$ Centers for Disease Control and Prevention, Atlanta, GA, USA. ${ }^{3}$ Noguchi Memorial Institute for Medical Research, University of Ghana, Accra, Ghana. ${ }^{4}$ University of Ghana, Accra, Ghana. ${ }^{5}$ Veterinary Services of Ghana, Accra, Ghana. ${ }^{6}$ Present Address: NAMRU-3, PSC 452, P.O Box 5000, FPO, AE 09835-99983A Imtidad Ramses Street. Adjacent to Abbassia Fever Hospital, Abbassia, Cairo, Egypt.

Received: 17 May 2016 Accepted: 22 June 2016

Published online: 08 July 2016

\section{References}

1. Gonzalez JP, Cornet JP, Wilson ML, Camicas JL. Crimean-Congo haemorrhagic fever virus replication in adult Hyalomma truncatum and Amblyomma variegatum ticks. Res Virol. 1991:142:483-488.

2. Chinikar $\mathrm{S}$ et al. Serological evaluation of Crimean-Congo hemorrhagic fever in humans with high-risk professions living in enzootic regions of Isfahan province of Iran and genetic analysis of circulating strains. Vector-Borne Zoonotic Dis. 2012;12:733-8.

3. Hoogstraal H. Review article: the epidemiology of tick-borne Crimean-Congo hemorrhagic fever in Asia, Europe, and africa. J Med Entomol. 1979;15:307-417.

4. Shepherd AJ, Swanepoel R, Cornel AJ, Mathee O. Experimental studies on the replication and transmission of Crimean-Congo hemorrhagic fever virus in some African tick species. Am J Trop Med Hyg. 1989:40:326-31.

5. Chisholm K et al. Crimean-Congo hemorrhagic fever virus in ticks from imported livestock. Egypt Emerg Infect Dis J. 2012:18:181.

6. Camicas J et al. Ecology of ticks as potential vectors of Crimean-Congo hemorrhagic fever virus in Senegal: epidemiological implications. Arch Virol. 1990;1:303-22

7. Saluzzo JF et al. Isolation of Crimean-Congo haemorrhagic fever and rift valley fever viruses in upper Volta. Lancet. 2015;323:1179.

8. Nemes Z, et al. Human Crimean-Congo Hemorrhagic Fever, Sénégal. Emerg Infect Dis. 2004;10:1881-1882.

9. Appannanavar SB, Mishra B. An update on Crimean Congo hemorrhagic fever. J Glob Infect Dis. 2011:3:285-92.

10. Lwande OW et al. Seroprevalence of Crimean Congo hemorrhagic fever virus in ljara District, Kenya. Vector-Borne Zoonotic Dis. 2012:12:727-32.

11. Sisman A. Epidemiologic features and risk factors of Crimean^|^ndash;congo hemorrhagic fever in Samsun Province, Turkey. J Epidemiol. 2013;23:95-102.

12. Ansari $\mathrm{H}$ et al. Crimean-Congo hemorrhagic fever and its relationship with climate factors in southeast Iran: a 13-year experience. J Infect Dev Ctries. 2014;8:749-57.

13. Kamboj A, Pathak H. Crimean-Congo hemorrhagic fever: a comprehensive review. Vet World. 2013:6:812-7.

14. Zavitsanou A, Babatsikou F, Koutis C. Crimean Congo hemorrhagic fever: an emerging tick-borne disease. Heal Sci J. 2009:3:10-8.

15. Fajs $L$ et al. Prevalence of Crimean-Congo hemorrhagic fever virus in healthy population, livestock and ticks in Kosovo. PLoS One. 2014;9:e110982.

16. Drosten $\mathrm{C}$ et al. Rapid detection and quantification of RNA of Ebola and Marburg viruses, Lassa virus, Crimean-Congo hemorrhagic fever virus, rift valley fever virus, dengue virus, and yellow fever virus by real-time reverse transcription-PCR. J Clin Microbiol. 2002;40:2323-30.

17. Crowder $C D$ et al. Genotypic variation and mixtures of Lyme Borrelia in Ixodes ticks from North America and Europe. PLoS One. 2010;5:1-9.

18. Garrison AR et al. Development of a TagMan minor groove binding protein assay for the detection and quantification of Crimean-Congo hemorrhagic fever virus. Am J Trop Med Hyg. 2007;77:514-20.

19. Stachurski F, Lancelot R. Footbath acaricide treatment to control cattle infestation by the tick Amblyomma variegatum. Med Vet Entomol. 2006;20:402-12.

20. Leblebicioglu $\mathrm{H}$ et al. International journal of infectious diseases consensus report : preventive measures for Crimean-Congo hemorrhagic fever during Eid-al-adha festival. Int J Infect Dis. 2015;38:9-15.

\section{Submit your next manuscript to BioMed Central and we will help you at every step:}

- We accept pre-submission inquiries

- Our selector tool helps you to find the most relevant journal

- We provide round the clock customer support

- Convenient online submission

- Thorough peer review

- Inclusion in PubMed and all major indexing services

- Maximum visibility for your research

Submit your manuscript at www.biomedcentral.com/submit
Biomed Central 